\title{
Sharp crossover from composite fermionization to phase separation in microscopic mixtures of ultracold bosons
}

\author{
M. A. Garcia-March, ${ }^{1,2, *}$ B. Juliá-Díaz, ${ }^{1}$ G. E. Astrakharchik, ${ }^{3}$ Th. Busch, ${ }^{2,4}$ J. Boronat, ${ }^{3}$ and A. Polls ${ }^{1}$ \\ ${ }^{1}$ Departament d'Estructura i Constituents de la Matèria, Universitat de Barcelona, 08028 Barcelona, Spain \\ ${ }^{2}$ Physics Department, University College Cork, Cork, Ireland \\ ${ }^{3}$ Departament de Física i Enginyeria Nuclear, Universitat Politècnica de Catalunya, Barcelona, Spain \\ ${ }^{4}$ Quantum Systems Unit, Okinawa Institute of Science and Technology Graduate University, Okinawa, Japan
}

(Received 12 July 2013; published 3 December 2013)

\begin{abstract}
We show that a two-component mixture of a few repulsively interacting ultracold atoms in a one-dimensional trap possesses very diverse quantum regimes and that the crossover between them can be induced by tuning the interactions in one of the species. Starting from the composite fermionization regime, in which the interactions between both components are large and neither gas is phase coherent, our results show that a phase-separated state can be reached by increasing the interaction in one of the species. In this regime, the weakly interacting component stays at the center of the trap and becomes almost fully phase coherent, while the strongly interacting one is expelled to the edges of the trap. The crossover is sharp, as can be witnessed in the system's energy and in the occupation of the lowest natural orbital of the weakly interacting species. We show that such a transition is a few-atom effect which disappears for a large population imbalance.
\end{abstract}

DOI: 10.1103/PhysRevA.88.063604

PACS number(s): 67.85.-d, 03.75.Hh, 03.75.Mn, 05.30.Jp

Ensembles of a few interacting ultracold trapped atoms constitute unique quantum systems. They can be exceptionally well isolated from the environment, minimizing the role of decoherence, and are perfect candidates for the study of states with strong quantum correlations. Moreover, they are extremely versatile, as precise control over the shape of the trapping potential and the atom-atom interactions is routinely available in current experiments. Since at these energy scales often all degrees of freedom other than the positions can be ignored, they provide a simple system which still shows a great diversity of phenomena [1,2]. Experimentally, the loading of a small number of fermionic or bosonic atoms into a single trap has been achieved [3], and mixtures have been realized in optical lattice potentials [4]. Also, the strongly correlated low-dimensional Tonks-Girardeau (TG) gas has been achieved experimentally, which also requires low densities [5]. These systems constitute a natural ground for studies of squeezing and entanglement, with applications, for example, in precision measurements [6,7], thus leading to the great interest in their experimental realization.

In this article, we predict a sharp crossover between two very different regimes in microscopic, two-component mixtures of repulsively interacting bosons at zero temperature, corresponding to different interaction strengths between atoms of the same and different species when trapped in a onedimensional (1D) trap. In the first regime the interactions between the atoms of different species are strong, while the interactions between the atoms of the same species are weak. This is the so-called composite fermionization limit $[8,9]$, which shows strong anticorrelations between unlike atoms, similar to the ones between like atoms in the standard TG gas [10]. Since the occupation number of the lowest natural orbital for each species, $\mathrm{A}$ and $\mathrm{B}$, scales more rapidly than $\sqrt{N_{\mathrm{A}, \mathrm{B}}}$ but more slowly than $N_{\mathrm{A}, \mathrm{B}}$, neither of them is fully Bose

\footnotetext{
*magarciamarch@ecm.ub.edu
}

condensed. The second limit is obtained when the interaction strength in one of the species (A) is increased to the same level as the interaction strength between the species, while keeping the other component (B) weakly interacting. Surprisingly, this leads to phase separation, with species B being located in the center of the trap and the occupation number of its lowest natural orbital tending to $N_{\mathrm{B}}$; i.e., species B becomes Bose condensed.

The transition between the two limits is sharp and can be observed in the one- and two-body correlation functions, as well as in the degree of condensation of species B. In its dynamical form, i.e., upon an increase in the interaction strength in the A component in a time-dependent manner, it also allows us to create highly correlated quantum states. It is worth noting that the well-known mixing-demixing condition for weakly interacting Bose mixtures [11] predicts phase separation in both limits, but not spatial localization, which is crucial to finding the crossover. However, here we consider a small number of particles in the strong correlation regime, for which a mean-field Gross-Pitaevskii treatment is not appropriate.

We consider a system consisting of a small number of atoms of two bosonic components trapped in a 1D harmonic potential, $V(x)=\frac{1}{2} m \omega^{2} x^{2}$. We assume that each component is represented by a different hyperfine state of the same atomic species, and therefore all atoms have the same mass $m=m_{\mathrm{A}}=$ $m_{\mathrm{B}}$. The Hamiltonian for a fixed number of atoms $N_{\mathrm{A}, \mathrm{B}}$ in each species can then be written as $\hat{H}=\hat{H}_{\mathrm{A}}+\hat{H}_{\mathrm{B}}+\hat{H}_{\mathrm{AB}}$, where

$$
\begin{aligned}
\hat{H}_{\mathrm{A}} & =\sum_{j=1}^{N_{\mathrm{A}}}\left[\frac{-\hbar^{2}}{2 m} \frac{\partial^{2}}{\partial x_{j}^{2}}+V\left(x_{j}\right)\right]+\sum_{j<j^{\prime}}^{N_{\mathrm{A} t}} v_{\mathrm{int}}^{\mathrm{A}}\left(x_{j}, x_{j^{\prime}}\right), \\
\hat{H}_{\mathrm{AB}} & =\sum_{j=1}^{N_{\mathrm{A}}} \sum_{j^{\prime}=1}^{N_{\mathrm{B}}} v_{\mathrm{int}}^{A B}\left(x_{j}, y_{j^{\prime}}\right) .
\end{aligned}
$$

The part of the Hamiltonian for $\mathrm{B}, \hat{H}_{\mathrm{B}}$, is like $\hat{H}_{\mathrm{A}}$ but for $\mathrm{B}$ atoms. At low temperatures all scattering 
processes between the atoms can be described by a contact interaction, $v_{\text {int }}^{\mathrm{A}}=g_{\mathrm{A}} \delta\left(x_{j}-x_{j^{\prime}}\right), \quad v_{\text {int }}^{\mathrm{B}}=g_{\mathrm{B}} \delta\left(y_{j}-y_{j^{\prime}}\right)$, and $v_{\text {int }}^{\mathrm{AB}}=g_{\mathrm{AB}} \delta\left(x_{i}-y_{j}\right)$, where $x_{j}$ and $y_{j}$ are the positions of the atoms in species $\mathrm{A}$ and $\mathrm{B}$, respectively. Here $g_{\mathrm{A}(\mathrm{B})}$ and $g_{\mathrm{AB}}$ are the 1D intra- and interspecies coupling constants, respectively. We assume that they are all positive, corresponding to repulsive interactions, and can be fine-tuned by means of Feshbach or confinement-induced resonances [12]. We use harmonic oscillator units in the following and scale all lengths in units of the oscillator length $a_{0}=\sqrt{\hbar /(m \omega)}$ and all energies in units of $\hbar \omega$.

The physics of such a mixture can be understood by making the following ansatz for the ground-state wave function,

$$
\begin{aligned}
\Psi(X, Y)= & \Phi(X) \Phi(Y) \prod_{j<k}^{N_{\mathrm{A}}}\left|x_{k}-x_{j}-a_{\mathrm{A}}\right| \\
& \times \prod_{j<k}^{N_{\mathrm{B}}}\left|y_{k}-y_{j}-a_{\mathrm{B}}\right| \prod_{j, k}^{N_{\mathrm{A}}, N_{\mathrm{B}}}\left|x_{k}-y_{j}-a_{\mathrm{AB}}\right| .
\end{aligned}
$$

Here $\Phi$ is a Gaussian function that describes the exact solution for noninteracting atoms in the harmonic trap, $\Phi(X)=\exp \left[-\sum x_{i}^{2} / 2\right]$, with $X=\left\{x_{i}\right\}$ and $Y=\left\{y_{i}\right\}$. The $1 \mathrm{D} s$-wave scattering lengths $a_{\sigma}$, with $\sigma=\mathrm{A}, \mathrm{B}$, and $\mathrm{AB}$, for the interactions between A-A, B-B, and A-B atoms, are related to the corresponding coupling constants as $g_{\sigma}=$ $-2 \hbar^{2} /\left(m a_{\sigma}\right)$. The crossover discussed in this article occurs when changing the correlations in the A component from weak (ideal gas) to strong (TG gas) in a system where component B is ideal, $g_{\mathrm{B}}=0$, and the correlations between the A and the B components are large, $g_{\mathrm{AB}} \rightarrow \infty$. In this situation the ansatz for the wave function reduces to

$$
\Psi=\Phi(X) \Phi(Y) \prod_{j<k}^{N_{\mathrm{A}}}\left|x_{k}-x_{j}-a_{\mathrm{A}}\right| \prod_{j, k}^{N_{\mathrm{A}}, N_{\mathrm{B}}}\left|x_{k}-y_{j}\right| .
$$

In the limit of weak correlations in the A component $\left(g_{\mathrm{A}} \rightarrow 0\right)$, the terms containing $a_{\mathrm{A}}$ drop out from Eq. (3) and the wave function has nodes only when two atoms of different species meet, as expected in the composite fermionization limit [8]. For strong correlations in the A component $\left(g_{\mathrm{A}} \rightarrow \infty\right)$, on the other hand, ansatz (3) gains additional nodes and the wave function vanishes whenever two atoms of species A-A or A-B meet. In this regime the system is a mixture of a TG gas (component A) and an ideal gas (component B), which we refer to as a TG-BEC gas. Wave function (3) is not exact, however, it correctly describes the physical properties of the system, as we discuss below. Finally, if all coupling constants are large the system falls into a family of exactly solvable models discussed in Ref. [13].

To characterize the transition from the composite fermionization limit to a TG-BEC gas when $g_{\mathrm{A}}$ is tuned from 0 to large values, in the following we discuss the quantum many-body correlations, the spatial localization behavior, and the coherence properties. For $N_{\mathrm{B}} \geqslant N_{\mathrm{A}}$ the TG-BEC gas is known to be phase separated [14], with the B component being located in the center of the trap and the A component occupying the outer regions. In this case the occupation of the lowest lying natural orbital for species B is of the order of $N_{\mathrm{B}}$, which implies that the component is Bose condensed and fully phase
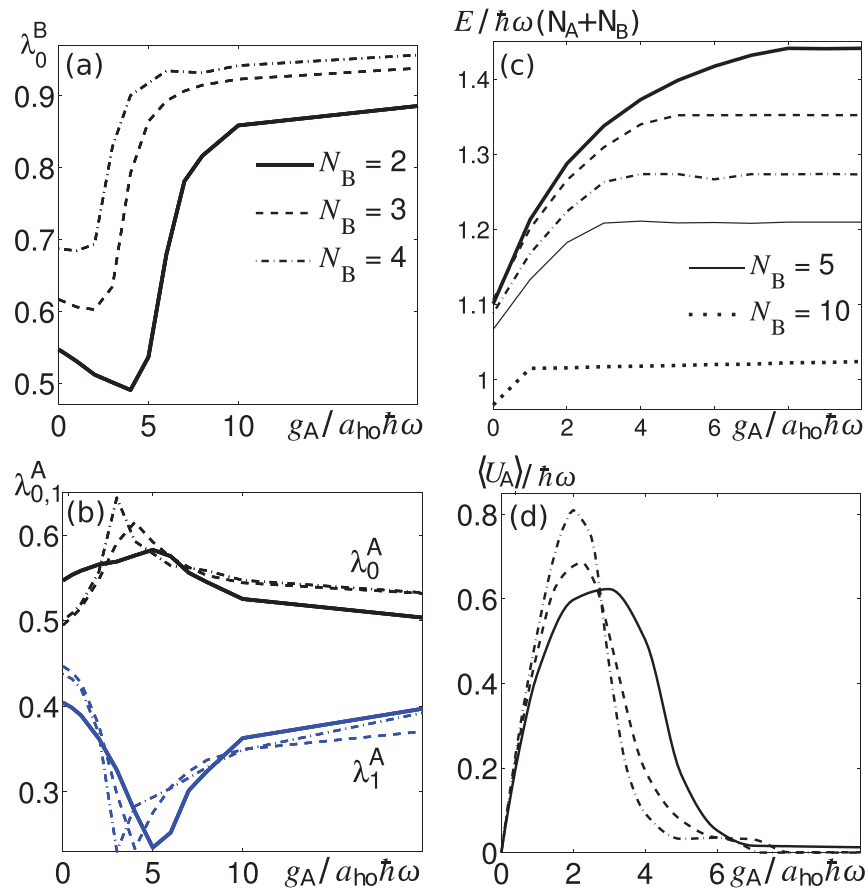

FIG. 1. (Color online) Occupations $\lambda_{0}$ and $\lambda_{1}$ of the lowest lying natural orbitals, energies per atom $E$, and interaction energies $\left\langle U_{A}\right\rangle$ as a function of the interaction strength $g_{\mathrm{A}}$ in the A component. (a) Natural orbital occupation numbers $\lambda_{0}$ for species B and (b) $\lambda_{0}$ (black lines) and $\lambda_{1}$ (blue lines) for species A. In (a) and (b) $N_{\mathrm{A}}=2$ and $N_{\mathrm{B}}=2,3,4$, and the results are calculated via direct diagonalization. (c) Energy per atom for $N_{\mathrm{B}}=2,3,4,5$, and 10, calculated using DMC. (d) Average interaction energy of species A, $\left\langle U_{\mathrm{A}}\right\rangle=\left\langle\sum_{j<j^{\prime}}^{N_{\mathrm{A}}} v_{\text {int }}^{A}\left(x_{j}, x_{j^{\prime}}\right)\right\rangle$. In all cases, $g_{\mathrm{B}}=0$ and $g_{\mathrm{AB}}$ is large.

coherent. Conversely, in the composite fermionization limit the phases are not separated and the occupation of a natural orbital for both species is smaller than $N_{\mathrm{A}, \mathrm{B}}$, indicating that none of the species is fully Bose condensed or localized in the center of the trap.

To calculate the ground-state properties we use direct diagonalization of the Hamiltonian [14] and the diffusion Monte Carlo (DMC) method [15]. The phase-separated TG-BEC is realized with $g_{\mathrm{B}}=0$ and $g_{\mathrm{AB}}=500 \hbar \omega a_{0}$ (diagonalization) or $g_{\mathrm{AB}} \rightarrow \infty$ (DMC). We calculate the one-body density matrix (OBDM), $\rho_{1}=\left\langle\hat{\Psi}^{\dagger}(x) \hat{\Psi}\left(x^{\prime}\right)\right\rangle$, and its diagonalization gives the natural orbitals and their occupation numbers $\lambda_{i}$. In Figs. 1(a) and 1(b), we show these occupation numbers for the lowest lying natural orbitals for systems of $N_{\mathrm{A}}=2$ and $N_{\mathrm{B}}=$ 2, 3, 4 atoms as a function of increasing A-A interactions. One can clearly see that the ground-state occupation for the ideal component, $\lambda_{0}^{\mathrm{B}}$, sharply increases between the composite fermionization and the phase-separated TG-BEC limits, while in the other component $\lambda_{0}^{\mathrm{A}}$ displays a change around the crossover point and settles to $\sim 0.5$ for large $g_{\mathrm{A}}$. The value range in which the crossover happens also overlaps with the point at which the energy per atom reaches a plateau [see Fig. 1(c)] . This point is characteristic for the number of atoms in the B component and decreases as $N_{\mathrm{B}}$ is increased. As long as $g_{\mathrm{A}}$ is small, the energy growth rate is similar for all cases, however, it saturates more rapidly for larger particle number imbalances, indicating that the crossover behavior 
only appears for small imbalances in the population of both components and, in this sense, is a purely microscopic effect.

The sharp crossover can also be observed by looking at the interaction energy $\left\langle U_{A}\right\rangle$ [see Fig. 1(d)], which vanishes in both the composite fermionization limit, because $g_{\mathrm{A}}=0$, and the TG-BEC limit, because $\rho_{2}^{A}\left(x_{1}, x_{1}\right)=0$. However, between these limits a maximum is visible, whose position approximately coincides with the position of the crossover. Since we are considering the zero-temperature case, this allows us to determine the order of the transition by considering the continuity of $d E / d g_{\mathrm{A}}$. Using the Hellmann-Feynman theorem [16] one finds that $g_{\mathrm{A}} d E / d g_{\mathrm{A}}=\left\langle\Psi\left|U_{A}\right| \Psi\right\rangle$, and since $\left\langle U_{\mathrm{A}}\right\rangle$ is continuous, the transition is a crossover.

Figure 1(c) shows the energies obtained using DMC, with ansatz (3) as a guiding function. In this method we are able to treat systems with larger particle numbers and we have checked that the energies calculated from direct diagonalization converge to those obtained with DMC as the number of modes is increased. An equally good agreement between the two methods is obtained for the calculations of the one- and two-body distribution functions discussed below.
To interpret the crossover observed in Fig. 1, let us first discuss the behavior of the single- and two-body correlations present in the system. The two-body distribution function (TBDF), $\rho_{2}$, for two atoms of the same species is defined as

$$
\rho_{2}^{A}\left(x_{1}, x_{2}\right)=N_{\mathrm{A}}\left(N_{\mathrm{A}}-1\right) \int d x_{3} \cdots d x_{N_{\mathrm{A}}} d y_{1} \cdots d y_{N_{\mathrm{B}}}|\Psi|^{2},
$$

and the cross two-body distribution function (CTBDF) for two atoms of different species is

$$
\rho_{2}^{A B}\left(x_{1}, y_{1}\right)=N_{\mathrm{A}} N_{\mathrm{B}} \int d x_{2} \cdots d x_{N_{\mathrm{A}}} d y_{2} \cdots d y_{N_{\mathrm{B}}}|\Psi|^{2} .
$$

Both distributions can be interpreted as the probability of finding two atoms at two well-defined positions, with the TBDF describing atoms of the same species and the CTBDF describing pairs made from unlike atoms.

In the composite fermionization limit $\left(g_{\mathrm{A}}=g_{\mathrm{B}}=0\right.$ and large $\left.g_{\mathrm{AB}}=500 \hbar \omega a_{0}\right)$, and for $N_{\mathrm{A}}=N_{\mathrm{B}}$, the OBDMs for both species are identical, as shown in Figs. 2(a) and 2(b). The diagonal elements for $x=x^{\prime}\left(y=y^{\prime}\right)$ correspond to the single-particle density, which is shown by the dashed line in
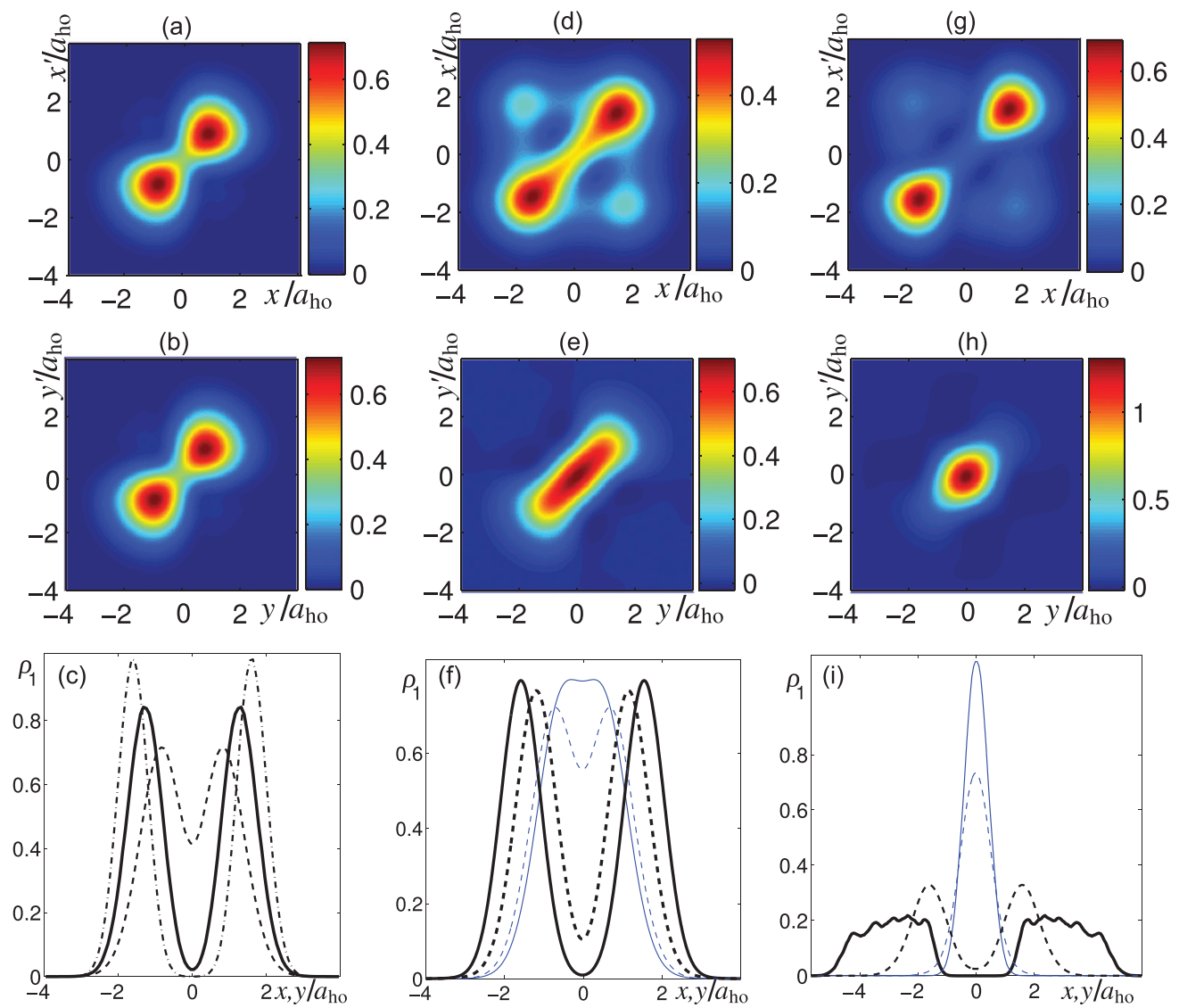

FIG. 2. (Color online) One-body density matrices $\rho_{1}\left(x, x^{\prime}\right)$ and single-particle densities in the composite fermionization and the phaseseparated TG-BEC limits. ( $\mathrm{a}, \mathrm{d}, \mathrm{g}$ ) OBDM for species A and (b, e, h) OBDM for species B, with $N_{\mathrm{A}}=N_{\mathrm{B}}=2$. (a, b) The system in the composite fermionization limit $\left(g_{\mathrm{A}}=0\right),(\mathrm{d}, \mathrm{e})$ an intermediate situation with $g_{\mathrm{A}}=5 \hbar \omega a_{0}$, and $(\mathrm{g}, \mathrm{h})$ the system in the phase-separated TG-BEC regime $\left(g_{\mathrm{A}}=7 \hbar \omega a_{0}\right)$. (c) Density profiles in the composite fermionization limit for $N_{\mathrm{A}}=N_{\mathrm{B}}=2,10$, and 20 (dashed, solid, and dash-dotted lines, respectively), calculated with DMC. Note that the densities for both species coincide. (f) Density profiles (species A, black; species B, blue) in the same limit for imbalanced systems with $N_{\mathrm{A}}=2$ and $N_{\mathrm{B}}=3,5$ (dashed and solid lines, respectively). (i) Density profiles in the phase separation limit, calculated with DMC, for $N_{\mathrm{A}}=N_{\mathrm{B}}=2$ and 10 (dashed and solid lines, respectively). For all situations $g_{\mathrm{B}}=0$ and $g_{\mathrm{AB}}$ is large. 

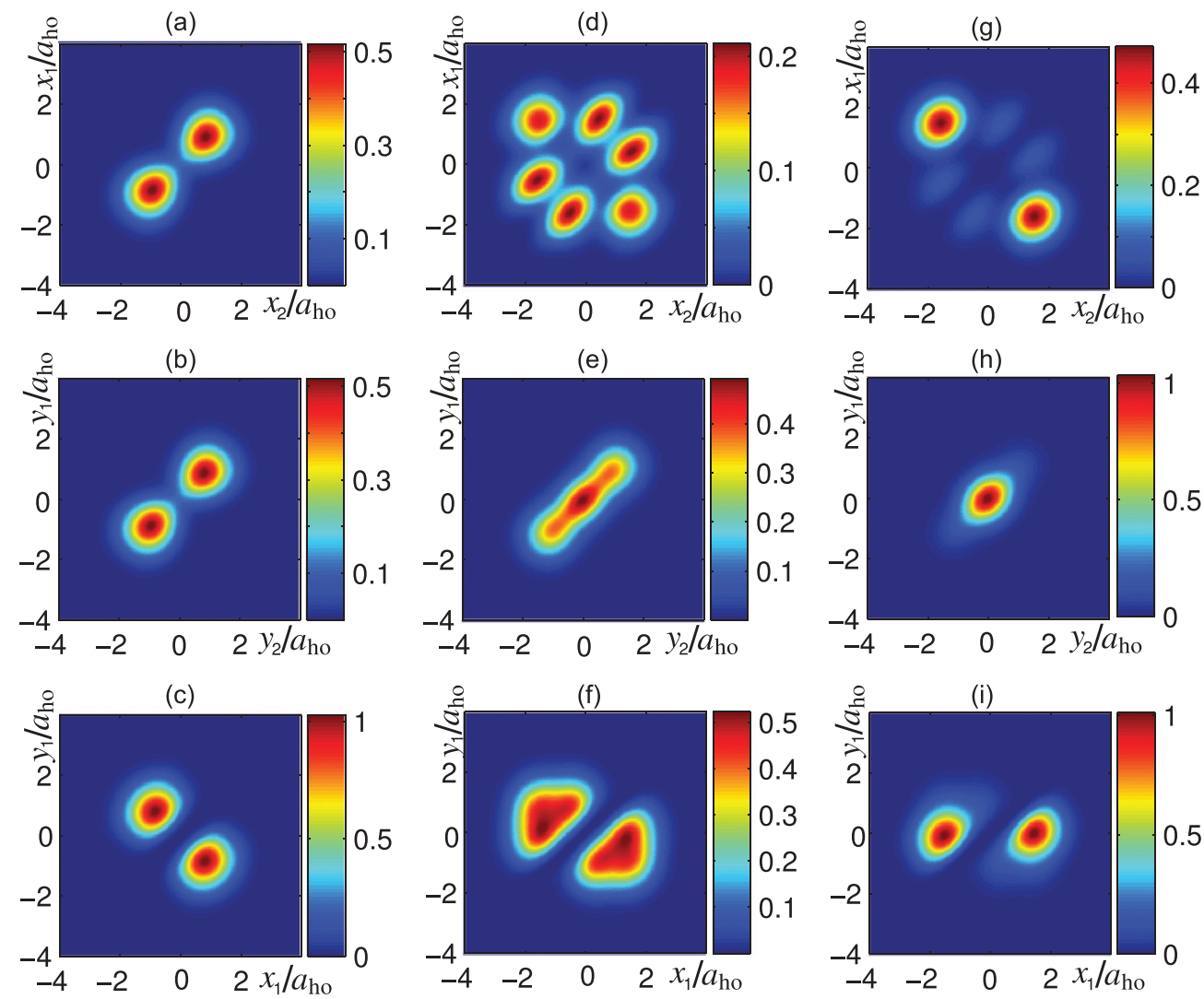

FIG. 3. (Color online) Two-body distribution functions in the composite fermionization and the phase-separated TG-BEC limits for a system with $N_{\mathrm{A}}=N_{\mathrm{B}}=2$. (a, d, g) TBDF for species A, (b, e, h) TBDF for species B, and (c, f, i) CTBDF. (a-c) The composite fermionization limit $\left(g_{\mathrm{A}}=0\right),(\mathrm{d}-\mathrm{f})$ an intermediate situation, $g_{\mathrm{A}}=5 \hbar \omega a_{0}$, and $(\mathrm{g}-\mathrm{i})$ the phase-separated TG-BEC limit $\left(g_{\mathrm{A}}=7 \hbar \omega a_{0}\right)$.

Fig. 2(c), and the occupation of the lowest lying natural orbital is $\lambda_{0}^{\mathrm{A}, \mathrm{B}} \simeq 0.55$ [see Figs. 1(a) and 1(b)]. The corresponding TBDFs for both species are shown in Figs. 3(a) and 3(b), and the finite values along the diagonal confirm that two atoms of the same species can occupy the same position. However, two atoms of different species cannot overlap, as shown by the vanishing CTBDF along the $x_{1}=y_{1}$ diagonal in Fig. 3(c). Furthermore, the absence of any probability in the equal-sign quarters in the CTBDF indicates that if an atom of species A is found on one side of the trap, all atoms of species B will be found on the other side. We note that wave function (3) also correctly reproduces the density and TBDFs (not shown). This behavior persists for larger, balanced numbers of atoms $\left(N_{\mathrm{A}}=\right.$ $N_{\mathrm{B}}$ ), with the two peaks in the density distribution moving farther apart due to the increased repulsive energies between the atoms in different species [see Fig. 2(c)]. However, in an unbalanced microscopic system with $N_{\mathrm{B}}>N_{\mathrm{A}}$, the OBDMs are not equal for both species, and the larger component has a tendency to occupy the center of the trap [see densities in Fig. 2(f)] and become fully phase coherent despite the strong interactions with the smaller component. This becomes more pronounced for larger imbalances and is shown in Fig. 1(a) by the growing occupation number of the lowest lying natural orbital for species B.

Increasing the correlations in the A component, the system evolves towards the TG-BEC limit and starts to phase separate [Figs. 2(d) and 2(e)]. For $g_{\mathrm{A}}=7 \hbar \omega a_{0}$ the spatial overlap between the two components is almost gone [Figs. 2(g) and 2(h)] and the occupation of the lowest lying natural orbital of species $\mathrm{B}$ has risen to $\lambda_{0}^{\mathrm{B}} \simeq 0.9$, indicating that this species is almost fully condensed. Due to the phase separation the correlations between the $\mathrm{A}$ and the $\mathrm{B}$ components are also reduced, which can be seen in the increased value of $\lambda_{0}^{A}$ for small, but finite values of $g_{A}$ in Fig. 1(a), however, with increasing correlations inside the A component, it moves into the TG regime and $\lambda_{0}^{\mathrm{A}}$ settles at $\simeq 0.5$ [see Fig. 1(b)]. The TBDF for $\mathrm{B}$ corresponds to a condensed cloud in the center of the trap [Fig. 3(h)], while that for A is indicative of a fragmented state of two single atoms on each side of B [Fig. 3(g)]. The CTBDF [see Fig. 3(i)] is consistent with this picture. A similar picture remains valid in larger, balanced systems [see Fig. 2(i), obtained with DMC for $g_{\mathrm{AB}} \rightarrow \infty$ and $g_{\mathrm{A}} \rightarrow \infty$ ] or in systems with a small atom number imbalance. In such cases, when species A is spatially separated it behaves as two TG gases occupying each side of $\mathrm{B}$, each with half of the atoms, which are anticorrelated [10].

For values of $g_{\mathrm{A}}$ approaching the crossover region, the densities of both components start to phase separate [see Figs. 2(d) and 2(e)] and the occupation of the lowest lying natural orbital of species B displays a small minimum before growing rapidly [see Fig. 1(a)]. Due to the enhanced correlations in the A component, the two A atoms start to avoid each other, which induces a minimum along the diagonal $x_{1}=x_{2}$ of the respective TBDF [see Fig. 3(d)]. The increased 
tendency toward phase separation also leads to the appearance of peaks in the other diagonal, which correspond to localization of one atom of species $A$ on each side of the trap. The TBDF of species B shows a maximum in the center of the trap, in agreement with the tendency of the atoms of this species to localize there [see Figs. 2(e) and 3(e)]. Since the interactions between the two species remain strong, the CTBDF [Fig. 3(f)] maintains the 0 along the diagonal $x_{1}=y_{1}$ in this regime. It also develops an increased probability around $y_{1}=0$ and $x_{1}$ finite, corresponding to a density of the phase-separated state with $\mathrm{B}$ atoms in the center and $\mathrm{A}$ atoms at the edges. This behavior persists for larger, balanced systems.

Tuning the interactions in the A component dynamically allows a transition in which the spatially localized A component is split into two parts by a barrier given by the B atoms. Such a system resembles a double-well setup for the A component, which is known to allow for interesting, nonclassical, many-atom superposition states between the two parts of the A component [17]. However, let us stress again that in this case the barrier is raised by increasing the interactions in the A component (not in species B, which forms the barrier) and stems from internal correlations in this species and between the two species.

In conclusion, we have studied in an exact way the groundstate properties of a small, trapped, 1D, two-component Bose gas, using a diagonalization method and a DMC approach. This has allowed us to identify a sharp crossover between the regimes of composite fermionization and phase separation, and we have shown that in the latter regime the strongly correlated component fragments into two parts around the weakly correlated one. We believe that this crossover is of key interest for upcoming experiments with small mixtures of interacting ultracold bosons. In addition, we have pointed out that this crossover can be used in a dynamical way to create a spatially strongly correlated many-atom state of the kind that is of interest in applications in quantum information. Our results are restricted to $1 \mathrm{D}$ systems, as neither the composite fermionization limit nor the TG gas limit is defined in higher dimensions.

Note added in proof. Recently, a work calculating the energies in the limits discussed here in an analytical way appeared [18].

This project was supported by Science Foundation Ireland under Project No. 10/IN.1/I2979. We also acknowledge partial financial support from DGI (Spain) Grants No. FIS201125275 and No. FIS2008-00784 (TOQATA) and Generalitat de Catalunya Grants No. 2009SGR-1003 and No. 2009SGR01289. G.E.A. and B.J.D. was supported by a fellowship from MEC (Spain) through the Ramón y Cajal program.
[1] D. Blume, Rep. Prog. Phys. 75, 046401 (2012).

[2] I. Bloch and W. Zwerger, Rev. Mod. Phys. 80, 885 (2008).

[3] X. He, P. Xu, J. Wang, and M. Zhan, Opt. Express. 18, 13586 (2010); F. Serwane, G. Zürn, T. Lompe, T. B. Ottenstein, A. N. Wenz, and S. Jochim, Science 332, 336 (2011); R. Bourgain, J. Pellegrino, A. Fuhrmanek, Y. R. P. Sortais, and A. Browaeys, arXiv:1305.3802.

[4] S. Will, T. Best, S. Braun, U. Schneider, and I. Bloch, Phys. Rev. Lett. 106, 115305 (2011).

[5] T. Kinoshita, T. Wenger, and D. S. Weiss, Science 305, 1125 (2004); B. Paredes, A. Widera, V. Murg, O. Mandel, S. Folling, J. I. Cirac, G. V. Shlyapnikov, T. W. Hänsch, and I. Bloch, Nature 429, 277 (2004); E. Haller, M. Gustavsson, M. J. Mark, J. G. Danzl, R. Hart, G. Pupillo, and H.-C. Nagerl, Science 325, 1224 (2009).

[6] C. Orzel, A. K. Tuchman, M. L. Fenselau, M. Yasuda, and M. A. Kasevich, Science 291, 2386 (2001); A. Itah, H. Veksler, O. Lahav, A. Blumkin, C. Moreno, C. Gordon, and J. Steinhauer, Phys. Rev. Lett. 104, 113001 (2010).

[7] J. Estève, C. Gross, A. Weller, S. Giovanazzi, and M. K. Oberthaler, Nature 455, 1216 (2008).
[8] S. Zöllner, H.-D. Meyer, and P. Schmelcher, Phys. Rev. A 78, 013629 (2008).

[9] O. E. Alon, A. I. Streltsov, and L. S. Cederbaum, Phys. Rev. A 76, 062501 (2007).

[10] M. D. Girardeau, E. M. Wright, and J. M. Triscari, Phys. Rev. A 63, 033601 (2001).

[11] D. S. Hall, M. R. Matthews, J. R. Ensher, C. E. Wieman, and E. A. Cornell, Phys. Rev. Lett. 81, 1539 (1998).

[12] M. Olshanii, Phys. Rev. Lett. 81, 938 (1998); E. Haller, M. J. Mark, R. Hart, J. G. Danzl, L. Reichsöllner, V. Melezhik, P. Schmelcher, and H.-C. Nägerl, ibid. 104, 153203 (2010).

[13] M. D. Girardeau and A. Minguzzi, Phys. Rev. Lett. 99, 230402 (2007).

[14] M. A. Garcia-March and Th. Busch, Phys. Rev. A 87, 063633 (2013).

[15] J. Boronat and J. Casulleras, Phys. Rev. B 49, 8920 (1994).

[16] K. V. Kheruntsyan, D. M. Gangardt, P. D. Drummond, and G. V. Shlyapnikov, Phys. Rev. Lett. 91, 040403 (2003).

[17] M. A. Garcia-March, D. R. Dounas-Frazer, and L. D. Carr, Phys. Rev. A 83, 043612 (2011); Front. Phys. 7, 131 (2012).

[18] N. T. Zinner, A. G. Volosniev, D. V. Fedorov, A. S. Jensen, and M. Valiente, arXiv:1309.7219. 